\title{
PARticipation in inflammation
}

\author{
Shaun R. Coughlin and Eric Camerer \\ Cardiovascular Research Institute, University of California at San Francisco, San Francisco, California, USA \\ J. Clin. Invest. 111:25-27 (2003). doi:10.1172/JCI200317564.
}

In this issue of the JCI, Ferrell and colleagues report that mice lacking the proteinase-activated receptor-2 (PAR-2) were protected against a form of adjuvant-induced arthritis (1). Herein we discuss protease-activated receptors, their mechanism of activation, and the contexts in which they are thought to function. A useful unifying hypothesis emerges: protease-activated receptors link tissue injury to appropriate cellular responses, and such responses, while normally homeostatic, may contribute to disease.

Protease-activated receptor-1 (PAR-1), the prototypical PAR, was identified in a search for the receptors that mediate the activation of platelets and other cells by the coagulation protease thrombin. Known to be a seven transmembrane domain G-protein-coupled receptor, PAR-1 is activated by proteolysis (Figure 1). Thrombin binds to PAR-1's N-terminal exodomain and cleaves it after Arg41 to generate a new receptor $\mathrm{N}$-terminus. The first six amino acids of this new N-terminus, SFLLRN, then serve as a tethered ligand that binds intramolecularly to the receptor's heptahelical bundle to effect transmembrane signaling and $G$ protein activation. Thus PARs are, in essence, peptide receptors that carry their own ligands, which lie hidden until revealed by receptor cleavage. The synthetic peptide SFLLRN, which

\footnotetext{
Address correspondence to: Shaun R. Coughlin, University of California at San Francisco, HSE-1300, 513 Parnassus Avenue, San Francisco, California 94143-0130, USA. Phone: (415) 476-6174; Fax: (415) 476-8173; E-mail: coughlin@cvrimail.ucsf.edu. Conflict of interest: The authors have declared that no conflict of interest exists. Nonstandard abbreviations used: proteaseactivated receptor (PAR); proteinase-activated receptor-2 (PAR-2).
}

mimics the PAR- 1 tethered ligand, is a PAR-1 agonist and activates PAR-1 independent of thrombin and receptor cleavage. Such peptide agonists have provided a ready tool for probing the effects of PAR activation in vitro and in vivo.

Four PARs are currently known (recent reviews include refs. 2-4). PAR-1, PAR-3, and PAR- 4 can be activated by thrombin, and thrombin is almost certainly a physiological activator of these receptors in vivo. Several other proteases can cleave these receptors productively and may also contribute to their function in vivo. PAR-2 can be activated by multiple trypsin-like serine proteases including: trypsin itself, mast cell tryptase, neutrophil proteinase 3 , tissue factor/factor VIIa/factor Xa, and membrane- tethered serine protease- 1 . However PAR-2 is not cleaved by thrombin.

\section{What roles might PARs play in normal physiology and disease?}

Because the connection between a specific PAR and a specific protease is best established for thrombin, this question is first considered in the context of PARs as detectors of coagulation proteases. Before launching into this conceit, it is important to state that the question of which proteases activate PARs in vivo has not been fully answered, and there will undoubtedly be more to the story.

Thrombin is the main effector protease of the coagulation cascade, a series of zymogen conversions that are triggered when coagulation factors in plasma contact tissue factor expressed

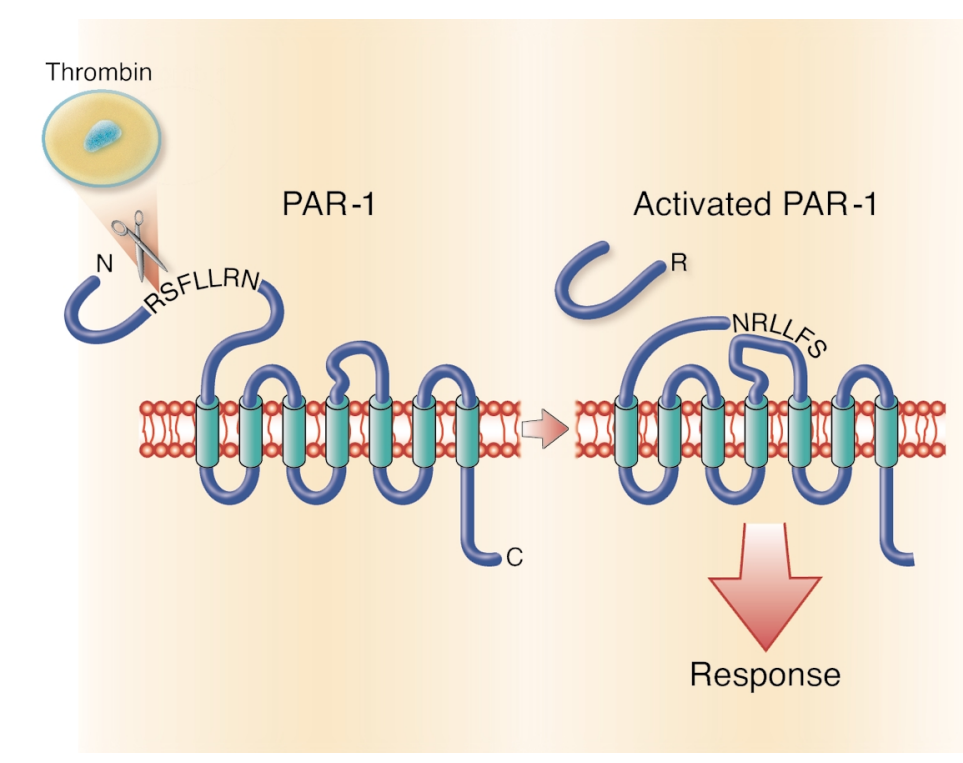

Figure 1

Mechanism of PAR-1 activation. The $\mathrm{N}$-terminus of PAR-1, a seven transmembrane domain $\mathrm{C}$ protein-coupled receptor, contains a protease cleavage site that, once cleaved by thrombin, results in a new $\mathrm{N}$-terminus. The new $\mathrm{N}$-terminal sequence, SFLLRN, acts as a tethered ligand and binds intramolecularly to the heptahelical body of the receptor to effect transmembrane signaling and $G$ protein activation. 
in the extravascular compartment or recruited to a site of injury. PARs are expressed by a variety of cell types in and around blood vessels (2), perhaps poised to detect activation of coagulation. PAR signaling largely accounts for the activation of platelets by thrombin, and such signaling plays a key role in hemostasis and thrombosis $(2,5,6)$. Thus a role for PARs in mediating cellular responses to injury at the level of platelets and hemostasis is clear. The known actions of PARs on other cell types suggest that this notion can be extended.

The set of responses to PAR- 1 activation in endothelial cells is concordant with the hypothesis that PARs help orchestrate a coordinated response to tissue injury (2). Many of the responses (mobilization of von Willebrand factor and P-selectin from Weibel-Palade bodies, expression of IL-8 and other chemokines as well as ICAM-1, VCAM-1 and other adhesion molecules, induction of COX-2 and production of prostanoids, plateletactivating factor and nitric oxide, changes in cell shape and monolayer permeability) presumably serve to recruit platelets and leukocytes to sites of injury and promote access of plasma proteins to the extravascular space. Other responses (expression of decay-accelerating factor and negative regulators of apoptosis) may serve protective roles. Still others (heparinbinding epidermal growth factor synthesis and shedding, PDGF and connective tissue growth factor production, and metalloproteinase and matrix production) may serve roles in blood vessel or tissue remodeling. Like PAR-1, PAR-2 is Gq-coupled, and endothelial cell responses to PAR-2 activation are very similar but apparently not identical to those elicited by PAR-1 (7). In smooth muscle cells and fibroblasts, PAR activation triggers growth factor and matrix production, migration, and proliferation responses envisioned to have a role in tissue remodeling. PARs are also expressed on sensory nerves, where their activation contributes to neurogenic edema and modulates nociception $(8,9)$. PAR-1 may also promote mast cell degranulation, directly or indirectly (10), and PAR-2 can respond to mast cell granzymes. These considerations suggest that together, the coagulation cascade and PARs provide a system that detects tissue injury (disruption of vascular integrity) and triggers a set of cellular responses that contribute to hemostasis, inflammation, tissue repair, and protection of the injured tissue through the perception of pain. Given this view of PARs as sensors of tissue injury and mediators of appropriate cellular responses, it is tempting to ask whether they might play a role in the decision to mount an adaptive immune response. Intriguingly, PAR expression has been reported in dendritic cells (11) and in T cells (12), but the functional significance of PARs in these cell types is unknown.

\section{PAR-2 activation}

How might PAR-2 fit into this picture given that it is not activated by thrombin? PAR-2 can be activated by coagulation proteases upstream of thrombin (tissue factor/VIIa complex, factor $\mathrm{Xa}$, and the cognate ternary complex), but whether PAR-2 senses activation of the coagulation cascade in vivo remains to be established. PAR-2 can also be activated by mast cell tryptase, and PAR-2-deficient mice are protected against paw hyperalgesia induced by a mast cell degranulating drug (9). PAR-2 can also be activated by the neutrophil granzyme proteinase-3 (13). Thus PAR-2 may sense tissue injury via granzymes released from mast cells and other leukocytes. Regardless of how it becomes activated, PAR-2 clearly can trigger inflammatory responses in vivo, consistent with the notion that it may sense and mediate responses to tissue injury.

\section{Role for PAR-2 in arthritis}

The schemes outlined above beg the question of whether PAR signaling makes an important contribution to inflammatory responses. We will focus on PAR-2. PAR-2-activating peptides can trigger edema, leukocyte rolling and firm adhesion to the vascular endothelium, tissue infiltration by neutrophils, and hyperalgesia $(2,3)$. Moreover, PAR-2 deficiency confers some protection against leukocyte rolling and margination that occurs upon exteriorization of the cremaster (14), hyperreactivity in allergic inflammation of the airway (15), allergic dermatitis (16), and hyperalgesia induced by formalin or mast cell degranulation (9). Ferrell et al. (1) extend this question to another model that involves adjuvant-mediated injury.

The key finding by Ferrell et al. is that PAR-2-deficient mice were protected against a form of arthritis triggered by direct intra-articular and peri-articular injection of CFA (1). Relatively few mice were studied to generate the key Figure $4 \mathrm{a}(n=6-8)$ and Table $1(n=3-7)$, but the difference in acute and chronic joint swelling and in cartilage destruction at 30 days after arthritis induction is striking and statistically significant. Moreover, Busso and colleagues have found that PAR-2deficiency may protect in a different, antigen-induced arthritis model $(\mathrm{N}$. Busso, unpublished observations). If such results prove robust and reproducible, they raise a number of questions of basic and therapeutic import.

Which protease(s) activates PAR-2? Joint biopsies from patients with rheumatoid arthritis stain for fibrin suggesting that coagulation is active in this setting. Moreover, the thrombin inhibitor hirudin is effective in a collagen-induced arthritis model (17). Thus, smoldering activation of the coagulation cascade may contribute to PAR-2 activation (perhaps by factor $\mathrm{Xa}$ ), but it is equally possible that granzymes from mast cells or other leukocytes or still other proteases function in this context.

In which cell type(s) and processes is PAR-2 important? Ferrel et al. show, utilizing a lacZ marker of PAR-2 expression, that PAR-2 is restricted to the vasculature in normal joints and is greatly induced with no apparent cell type or anatomic specificity in the inflamed synovium. An inflamed wildtype joint as a specificity control might have been useful in this case. Is endothelial PAR-2 playing a role in recruiting monocyte/macrophages, neutrophils, and T cells? Is PAR-2 on leukocytes contributing to their activation? Is PAR-2 in neurons contributing to edema? These questions can in principle be addressed in mouse models.

Lastly, to what extent does the specific model utilized by Ferrell and colleagues (1) relate to rheumatoid arthritis? Can lessons learned in the mouse regarding the importance of individual PARs (and PARs in general) in inflammation be applied directly to man, and is PAR-2 a rational target for anti-inflammatory therapy? With respect to the latter, it is important to 
note that, while the majority of reports cast PAR-2 as pro-inflammatory, PAR-2 agonists have been reported to be beneficial in several mouse models that involve inflammation or ischemia (18-20). Clearly there is much more to be learned regarding the biochemistry of PAR-2 activation and its roles in vivo. The report by Ferrell and colleagues will undoubtedly stimulate interest, and we look forward to answers to the questions posed above. They may well be more interesting than anything presently envisioned.

\section{Acknowledgments}

We thank Nathalie Busso, Laboratoire de Rhumatologie, Centre Universitaire Vaudois, Lausanne, Switzerland, for permission to mention her unpublished results.

1. Ferrell, W.R., et al. 2003. Essential role for proteinase activated receptor-2 in arthritis. J. Clin. Invest. 111:35-41. doi:10.1172/JCI200316913.

2. Coughlin, S.R. 2000. Thrombin signaling and protease-activated receptors. Nature. 407:258-264.
3. Vergnolle, N., Wallace, J.L., Bunnett, N.W., and Hollenberg, M.D. 2001. Protease-activated receptors in inflammation, neuronal signaling and pain. Trends. Pharmacol. Sci. 22:146-152.

4. Fiorucci, S., and Distrutti, E. 2002. Role of PAR2 in pain and inflammation. Trends. Pharmacol. Sci. 23:153-155.

5. Weiss, E.J., Hamilton, J.R., Lease, K.E., and Coughlin, S.R. 2002. Protection against thrombosis in mice lacking PAR3. Blood. 100:3240-3244.

6. Cook, J.J., et al. 1995. An antibody against the exosite of the cloned thrombin receptor inhibits experimental arterial thrombosis in the African green monkey. Circulation. 91:2961-2971.

7. Riewald, M., Petrovan, R.J., Donner, A., Mueller, B.M., and Ruf, W. 2002. Activation of endothelial cell protease activated receptor 1 by the protein C pathway. Science. 296:1880-1882.

8. Steinhoff, M., et al. 2000. Agonists of proteinase-activated receptor 2 induce inflammation by a neurogenic mechanism. Nat. Med. 6:151-158.

9. Vergnolle, N., et al. 2001. Proteinase-activated receptor-2 and hyperalgesia: a novel pain pathway. Nat. Med. 7:821-826.

10. Cirino, G., et al. 1996. Thrombin functions as an inflammatory mediator through activation of its receptor. J. Exp. Med. 183:821-827.

11. Steinhoff, M., et al. 1999. Proteinase-activated receptor-2 in human skin: tissue distribution and activation of keratinocytes by mast cell tryptase. Exp. Dermatol. 8:282-294.

12. Mari, B., et al. 1994. Thrombin and thrombin receptor agonist peptide induce early events of $\mathrm{T}$ cell activation and synergize with TCR cross linking for CD69 expression and interleukin 2 production. J. Biol. Chem. 269:8517-8523.

13. Uehara, A., Sugawara, S., Muramoto, K., and Takada, H. 2002. Activation of human oral epithelial cells by neutrophil proteinase 3 through protease-activated receptor-2. J. Immunol. 169:4594-4603.

14. Lindner, J.R., et al. 2000. Delayed onset of inflammation in protease-activated receptor-2deficient mice. J. Immunol. 165:6504-6510.

15. Schmidlin, F., et al. 2002. Protease-activated receptor 2 mediates eosinophil infiltration and hyperreactivity in allergic inflammation of the airway. J. Immunol. 169:5315-5321.

16. Kawagoe, J., et al. 2002. Effect of protease-activated receptor- 2 deficiency on allergic dermatitis in the mouse ear. Jpn. J. Pharmacol. 88:77-84.

17. Marty, I., et al. 2001. Amelioration of collageninduced arthritis by thrombin inhibition. J. Clin. Invest. 107:631-640.

18. Fiorucci, S., et al. 2001. Proteinase-activated receptor 2 is an anti-inflammatory signal for colonic lamina propria lymphocytes in a mouse model of colitis. Proc. Natl. Acad. Sci. USA. 98:13936-13941.

19. Milia, A.F., et al. 2002. Protease-activated receptor-2 stimulates angiogenesis and accelerates hemodynamic recovery in a mouse model of hindlimb ischemia. Circ. Res. 91:346-352.

20. Cocks, T.M., et al. 1999. A protective role for protease-activated receptors in the airways Nature. 398:156-160. 